\title{
ARTICLE
}

Received 26 Oct 2013 | Accepted 19 May 2014 | Published 23 Jun 2014

DOI: $10.1038 /$ ncomms5160

OPEN

\section{A cochlear-bone wave can yield a hearing sensation as well as otoacoustic emission}

Tatjana Tchumatchenko ${ }^{1} \&$ Tobias Reichenbach ${ }^{2}$

A hearing sensation arises when the elastic basilar membrane inside the cochlea vibrates. The basilar membrane is typically set into motion through airborne sound that displaces the middle ear and induces a pressure difference across the membrane. A second, alternative pathway exists, however: stimulation of the cochlear bone vibrates the basilar membrane as well. This pathway, referred to as bone conduction, is increasingly used in headphones that bypass the ear canal and the middle ear. Furthermore, otoacoustic emissions, sounds generated inside the cochlea and emitted therefrom, may not involve the usual wave on the basilar membrane, suggesting that additional cochlear structures are involved in their propagation. Here we describe a novel propagation mode within the cochlea that emerges through deformation of the cochlear bone. Through a mathematical and computational approach we demonstrate that this propagation mode can explain bone conduction as well as numerous properties of otoacoustic emissions.

\footnotetext{
${ }^{1}$ Theory of Neural Dynamics Group, Max Planck Institute for Brain Research, Max-von-Laue Strasse 4, 60438 Frankfurt am Main, Germany. ${ }^{2}$ Department of Bioengineering, Imperial College London, South Kensington Campus, London SW7 2AZ, UK. Correspondence and requests for materials should be addressed to T.R. (email: reichenbach@imperial.ac.uk).
} 
T he mammalian cochlea is an intricate device that acts as a spatial frequency separator ${ }^{1-4}$. Airborne sound vibrates the middle ear and evokes a pressure signal at the base of the fluid-filled inner ear (Fig. 1). The pressure oscillation then propagates as a surface wave on the basilar membrane, an elastic structure that separates two fluid-filled compartments in the cochlea. Different frequency components become spatially separated because, through changes in its material properties, the basilar membrane is tuned to a range of frequencies that systematically vary between the apical and the basal end. A segment of the basilar membrane near the base resonates at a high frequency, and segments from further apical positions resonate at successively lower frequencies. The wave on the basilar membrane elicited by a single frequency greatly increases in amplitude upon approaching its resonant position, beyond which it sharply declines ${ }^{2,4}$. A tonotopic map emerges in which high frequencies are detected near the base and low frequencies near the apex of the cochlea.

The basilar-membrane waves produced by different frequencies, however, do not simply superpose linearly. Instead, the basilar membrane at a given cochlear position responds nonlinearly to forcing near the resonant frequency of that location $^{3,4}$. The nonlinearity arises from mechanical activity of hair cells that reside on the basilar membrane. These cells can produce mechanical forces that greatly amplify weak stimuli; large vibrations are amplified less. The relation between the amplitude of the applied force and the resulting vibration is hence compressively nonlinear, and indicates that each basilarmembrane segment operates near a dynamic instability (Hopf bifurcation $)^{5-7}$.

The nonlinear response of the basilar membrane produces distortion when multiple pure tones are presented simultaneously ${ }^{8-11}$. As an example, a cubic nonlinearity yields a response at frequencies such as $2 f_{1}-f_{2}$ or $2 f_{2}-f_{1}$ when stimulated at two frequencies $f_{1}$ and $f_{2}$. Such distortion products indeed arise prominently in the cochlea. As they can not only be measured as basilar-membrane vibration but also with a microphone placed in the ear canal, they must be emitted from the cochlea into the ear canal. One accordingly refers to these tones as distortion-product otoacoustic emissions.

For a given frequency, the peak of the travelling wave is relatively sharp, with a longitudinal extent of only $\sim 0.5 \mathrm{~mm}$ (refs 3,4$)$. The cubic distortion frequencies $2 f_{1}-f_{2}$ or $2 f_{2}-f_{1}$, for instance, are therefore only created at a significant amplitude a

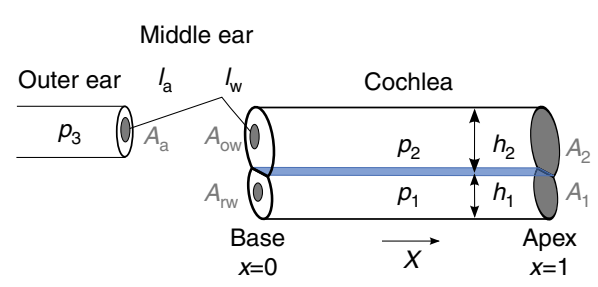

b

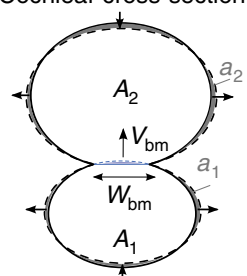

Cochlear cross-section

Figure 1 | Anatomy of the ear. (a) Sound causes a pressure vibration $p_{3}$ in the ear canal and a motion of the ear drum (area $A_{a}$ ). The middle ear's ossicles, namely the mallus of length $l_{\mathrm{a}}$, incus of length $I_{\mathrm{w}}$, and stapes convey the motion to the inner ear, or cochlea, to vibrate the oval window (area $A_{\text {ow }}$ ) and the round window (area $A_{r w}$ ). The pressures $p_{1}$ in the scala tympani and $p_{2}$ in the scala vestibuli change accordingly. (b) A transverse section of the inner ear shows the basilar membrane separating two chambers of cross-sectional area $A_{1}$ and $A_{2}$. Vibration of the membrane (velocity $V_{\mathrm{bm}}$ ) and deformation of the cochlear bone, at constant circumference, lead to area changes $a_{1}$ and $a_{2}$. when the two primaries $f_{1}$ and $f_{2}$ are sufficiently close, such that the corresponding peak regions overlap. The distortion hence arises from a narrow cochlear region from which it must propagate back to the base to cause a sound signal in the ear canal.

How the backward propagation occurs is currently intensely debated. Experiments show that distortion-product otoacoustic emissions consist of two components that differ in the temporal delay between their generation and the resulting emission in the ear canal ${ }^{12,13}$. One component has a long delay of a few milliseconds, whereas the delay of the other component is much shorter. The delay is measured through the change in phase of an emission upon altering the primary frequencies.

Some theoretical studies have suggested that both components emerge through waves on the basilar membrane that propagate backward from their generation site to the cochlear base $e^{14-16}$. Measurements of the intracochlear pressures as well as the cochlear microphonic potential support such reverse basilarmembrane waves ${ }^{17,18}$. Recent experimental measurements that have directly recorded the waves propagating along the membrane, however, only found forward-travelling waves, both at the primary frequencies as well as at the distortions ${ }^{19-21}$. Moreover, the stapes appear to vibrate at the distortion signal before the basilar membrane.

Recently we have proposed that the long-delay component of a distortion-product otoacoustic emission arises through waves on Reissner's membrane, another elastic membrane within the cochlea that extends in parallel to the basilar membrane from the cochlear base to the apex ${ }^{22}$. Our theoretical and numerical considerations show that short surface waves can propagate along Reissner's membrane, and that those waves can be created through the cochlear active process. Laser-interferometric measurements performed by ourselves have confirmed that such waves on Reissner's membrane exist and can arise from distortion on the basilar membrane.

As waves on Reissner's membrane have relatively short wavelengths, below $0.5 \mathrm{~mm}$ for frequencies above a few $\mathrm{kHz}$, such backward-propagating waves have slow speeds of a few metre per second. Distortion products emerging through those waves yield accordingly delays of a few milliseconds when propagating from their generation region to the middle ear.

How the short-delay component of an otoacoustic emission emerges, if not through backward waves on the basilar membrane, remains elusive. It has been suggested that compressional waves may transport a distortion signal within the cochlea ${ }^{19-21}$. Indeed, such waves can propagate in the cochlear fluids at large wavelengths and speeds. Because they involve no pressure difference across the basilar membrane and hence no membrane vibration, however, they cannot be produced by haircell forces acting on the membrane. Instead, their generation would require the active process to produces local volume changes, which have not yet been detected.

The mechanism of signal transmission in bone conduction remain similarly elusive. Bone conduction refers to our ability of hearing auditory signals through vibration of the cochlear bone, even in the absence of a functional middle ear ${ }^{23}$. Already one of the pioneers of hearing research, Békés $y^{24}$ conducted experiments in which he showed that the hearing sensation that is produced through bone conduction can be cancelled by stimulating the ear by an identical, but airborne, signal when its amplitude and phase are chosen carefully. Bone conduction hence appears to elicit the same basilar-membrane wave as is produced by airborne sound. This way of stimulating the ear is now increasingly used for constructing bone-conduction headphones, such as in the novel Google Glass device, that vibrate the cochlear bone and do not obstruct the ear canal. Such headphones allow to listen to 
environmental sound and, for example, additional information such as navigational directions that are inaudible to others. Despite the increasing use of this technology, we lack an understanding of how the cochlear bone vibration leads to basilar-membrane waves and hence the hearing sensation.

Early studies by Békésy ${ }^{24}$ as well as Herzog and $\mathrm{Krainz}^{25}$ suggested that the cochlear bone may not just vibrate homogeneously but deform under sound vibration. If the basilar membrane was not positioned in the middle of the cochlea, bone deformation could deflect the membrane and hence elicit the well-known basilar-membrane wave.

In this article we employ a cochlear model to show that deformation of the bone produces a wave that travels along the cochlea. Through mathematical and numerical methods we find that the deformation of the cochlear bone can evoke a traveling wave on the basilar membrane as well, and hence elicit a hearing sensation. We also show that otoacoustic emissions can emerge from the inner ear through the cochlear-bone wave. These emissions then have short delays of less than one millisecond, as observed for one component of otoacoustic emissions.

\section{Results}

Fluid dynamics. We start from a one-dimensional model of the inner ear (Fig. 1, model parameters are given in Table 1). The basilar membrane extends in the longitudinal $x$ direction and delineates two chambers. The one below the membrane is the scala tympani. We denote a pressure deviation therein from the resting pressure by $p_{1}$, a longitudinal fluid flow by $j_{1}$ and the cross-sectional area by $A_{1}$. The upper chamber comprises the scala media and scala vestibuli; this chamber's pressure deviation is $p_{2}$, its longitudinal fluid flow $j_{2}$ and its cross-sectional area $A_{2}$.

The longitudinal fluid flows in the upper and lower chamber carry momenta of $\rho \partial_{t} j_{1}$ and $\rho \partial_{t} j_{2}$, respectively, which must result from a longitudinal pressure gradient in that chamber:

$$
\begin{aligned}
& \rho \partial_{t} j_{1}=-A_{1} \partial_{x} p_{1}, \\
& \rho \partial_{t} j_{2}=-A_{2} \partial_{x} p_{2} .
\end{aligned}
$$

Here $\rho$ denotes the fluid density. The continuity equation states that a gradient in the longitudinal fluid flow of either chamber can only arise from a temporal change in the chamber's cross-sectional area or from a change in the fluid's density $\rho_{1 / 2}$. We denote by $a_{1}$ and $a_{2}$ the area change in the lower and the upper chamber, respectively. The total cross-sectional area of the respective chamber is $A_{1 / 2}+a_{1 / 2}$. We then find

$$
\begin{aligned}
& \partial_{x} j_{1}+\partial_{t} a_{1}+\frac{A_{1}}{\rho_{1}} \partial_{t} \rho_{1}=0, \\
& \partial_{x} j_{2}+\partial_{t} a_{2}+\frac{A_{2}}{\rho_{2}} \partial_{t} \rho_{2}=0 .
\end{aligned}
$$

A deviation in the fluid's density from its resting value $\rho_{0}$ is caused by a change in pressure through the fluid's compressibility $\kappa: \partial_{t} \rho_{1 / 2}=\rho_{0} \kappa \partial_{t} p_{1 / 2}$.

The cross-sectional area of either cochlear chamber can change because of basilar-membrane vibration (Fig. 1b). We assume that the membrane's cross-section deforms parabolically, with a midpoint velocity $V_{\mathrm{bm}}$ that is defined such that an upward membrane motion yields a positive velocity (Methods). This motion hence expands the lower chamber and shrinks the upper one:

$$
\partial_{t} a_{1}=-\partial_{t} a_{2}=\frac{2}{3} \cdot w_{\mathrm{bm}} \cdot V_{\mathrm{bm}}
$$

in which $w_{\mathrm{bm}}$ denotes the membrane's width. In the following we consider a sound signal at a single angular frequency $\omega$. Pressure vibration occurs at that same frequency, and we make an ansatz in which it propagates longitudinally with a wave vector $k$ and an amplitude $\tilde{p}_{1 / 2}$ :

$$
p_{1 / 2}=\tilde{p}_{1 / 2} e^{i \omega t-i k x}+\text { c.c. }
$$

Here c.c. denotes the complex conjugate. Similarly, the basilarmembrane velocity oscillates at frequency $\omega$ and propagates longitudinally, it can hence be written as:

$$
V_{\mathrm{bm}}=\tilde{V}_{\mathrm{bm}} e^{i \omega t-i k x}+\text { c.c. }
$$

We can now relate the difference of the pressure amplitudes across the basilar membrane to the vibrational amplitude that it evokes:

$$
\tilde{p}_{1}-\tilde{p}_{2}=Z_{\mathrm{bm}} \cdot \tilde{V}_{\mathrm{bm}} \cdot
$$

The coefficient $Z_{\mathrm{bm}}$ denotes the local acoustic impedance of the membrane, which in general depends on the frequency of

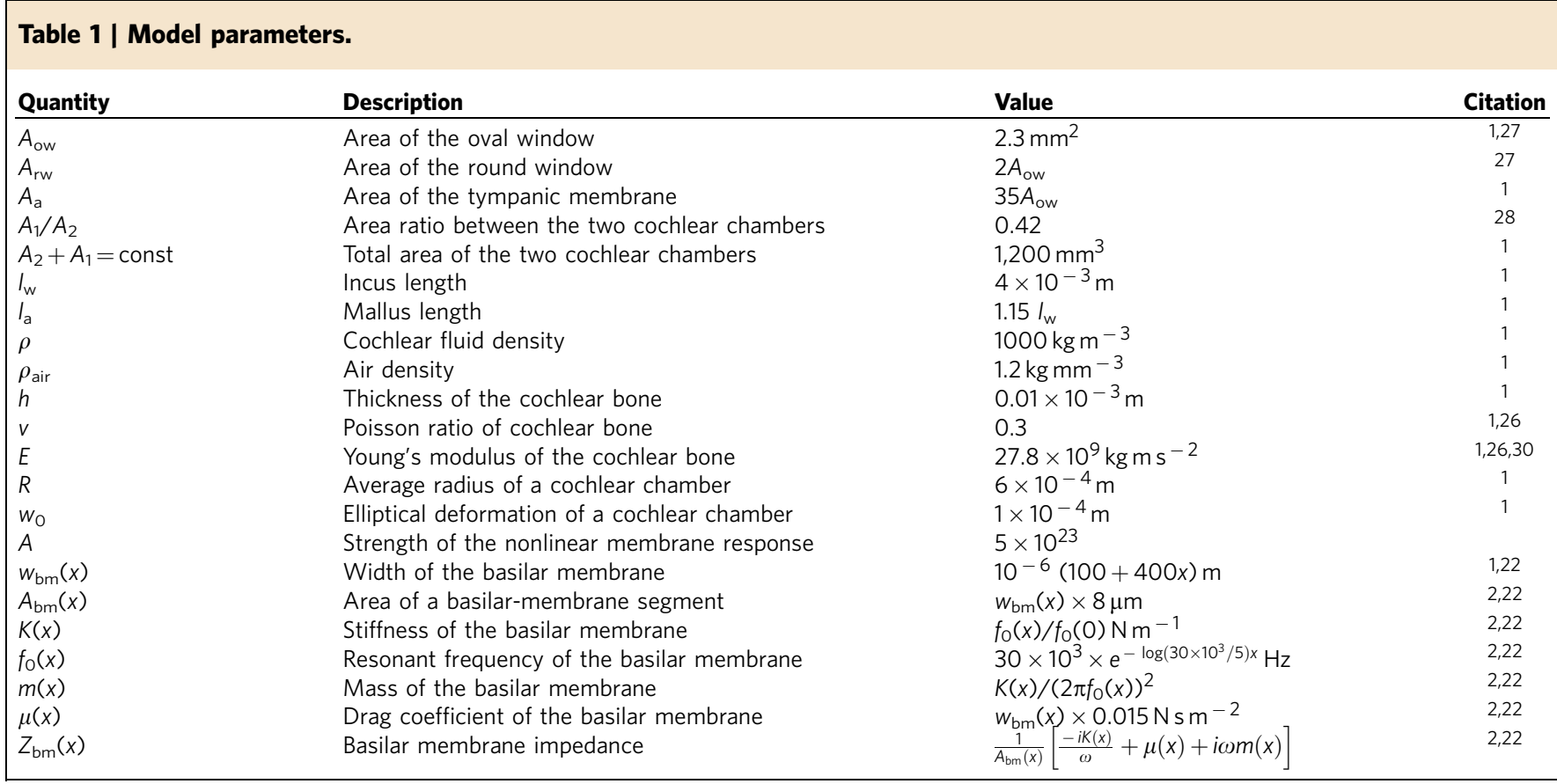


stimulation (Table 1). The equation (1) of momentum together with the equation (2) of continuity and equations (3) and (6) for the basilar-membrane velocity yield the well-known cochlear waves that propagate along the basilar membrane.

Here, we also include the possibility that the cochlear bone around the upper and lower chamber can be deformed through the intra-chamber pressure. Two types of deformation of a given cross-section are conceivable. First, the circumference of a crosssection may change. This requires compressibility of the chamber's wall. Second, the circumference may remain constant but the shape of the cross-section may vary. As the elastic modulus of bone is high, the first type of deformation has a much higher impedance than the second ${ }^{26,27}$. We hence only consider a deformation of the second type.

Which change in cross-sectional area results from a deformation that leaves the circumference constant? Let us approximate each chamber's cross-section by an ellipse-like shape that is deformed under internal pressure (Fig. $1 \mathrm{~b}$ and section on the linear-response coefficient $\mathrm{C}$ (Methods)). As a pressure change produces an equal force at every angle, no deformation can result when the cross section is circular. However, an asymmetric ellipse-like object that lacks rotational symmetry will deform under a pressure change. The impedance associated with this deformation has been studied in the literature ${ }^{26}$. Specifically, a decreasing internal pressure will increase the asphericity of the ellipse-like shape because, at constant circumference, the area is the smaller the more aspherical it is. Conversely, an enhanced pressure will tend to increase the cross-sectional area, which will hence deform towards a circle. For small deformations as we consider here, the area change depends linearly on the pressure deviation. The total change $a_{1 / 2}$ of the cross-sectional area of the upper respectively the lower chamber is hence the sum of one contribution from the membrane deflection and another contribution from the bone deformation:

$$
\begin{gathered}
\tilde{a_{1}}=-\frac{2 i}{3 \omega} \times w_{\mathrm{bm}} \times \tilde{V}_{\mathrm{bm}}+C \times \tilde{p}_{1}, \\
\widetilde{a_{2}}=\frac{2 i}{3 \omega} \times w_{\mathrm{bm}} \times \tilde{V}_{\mathrm{bm}}+C \times \tilde{p}_{2} .
\end{gathered}
$$

$C$ is a linear-response coefficient that we assume to be identical for both chambers. Its value can be derived by considering the elastic deformation of a tube (section on the linear-response coefficient C (Methods) as well as refs 26,28-30) and is given by

$$
C=\frac{4 \pi\left(1-v^{2}\right)}{E} \frac{R^{3} w_{0}^{2}}{h^{3}}
$$

Here, $E$ denotes the Young's modulus of the cochlear bone, $v$ the Poisson ratio, $h$ the thickness of the cochlear bone, $R$ the average radius of a chamber and $w_{0}$ the (approximately elliptical) deformation of the cross-sectional shape (see Table 1).

The fluid-momentum equation (1) with the continuity equation (2) as well as equations (6) and (7) for area and membrane vibration yield the matrix equation

$$
k^{2}\left(\begin{array}{l}
\tilde{p}_{1} \\
\tilde{p}_{2}
\end{array}\right)=\mathcal{M}\left(\begin{array}{l}
\tilde{p}_{1} \\
\tilde{p}_{2}
\end{array}\right)
$$

with the $2 \times 2$ matrix

$$
\mathcal{M}=-\omega \rho_{0}\left(\begin{array}{cc}
\frac{2 i w_{\mathrm{bm}}}{3 A_{1} Z_{\mathrm{bm}}}-\frac{\omega C}{A_{1}}-\omega \kappa & -\frac{2 i w_{\mathrm{bm}}}{3 A_{1} Z_{\mathrm{bm}}} \\
-\frac{2 i w_{\mathrm{bm}}}{3 A_{2} Z_{\mathrm{bm}}} & \frac{2 i w_{\mathrm{bm}}}{3 A_{2} Z_{\mathrm{bm}}}-\frac{\omega C}{A_{2}}-\omega \kappa
\end{array}\right) .
$$

The possible wave vectors $k$ hence follow from the eigenvalues of the matrix $\mathcal{M}$. The eigenvectors describe how the pressures in the upper and lower chamber relate to each other in the corresponding wave mode.

The eigenvalues and eigenvectors can be readily interpreted, as the different terms in the matrix $\mathcal{M}$ are of different orders of magnitude: $\left|w_{\mathrm{bm}} /\left(A_{1 / 2} Z_{\mathrm{bm}}\right)\right| \gg\left|\omega C / A_{1 / 2}\right| \gg \omega \kappa$. The basilar a

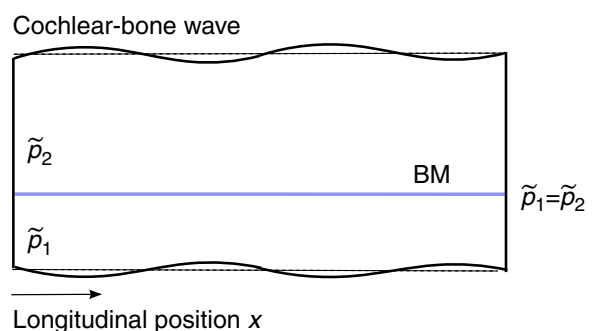

b

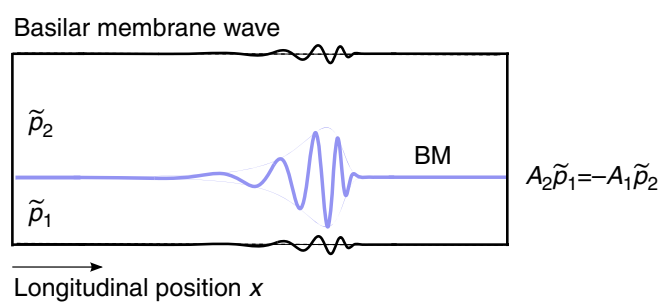

Figure 2 | Basilar-membrane and cochlear-bone wave. Two independent propagation modes, the basilar-membrane wave and the cochlear-bone wave, exist in a cochlea with a deformable bone. (a) Deformation of the cochlear bone propagates longitudinally as a wave that elicits, at a given location, identical pressure changes in both chambers and hence no vibration of the basilar membrane. (b) The basilar-membrane wave is evoked by a pressure difference across it. Because of the pressure changes in each chamber, this wave is accompanied by deformation of the cochlear bone.

membrane is significantly floppier than the cochlear bone, and yields a dominating contribution in the matrix $\mathcal{M}$. The effect of the fluid's compressibility is negligible. In the following sections we hence regard the fluid as incompressible.

As the matrix equation (9) has two degrees of freedom, there exist two eigenvectors that correspond to two distinct wave modes (Fig. 2). First, one eigenvector involves opposite pressures in the two chambers, $A_{2} \tilde{p}_{1}=-A_{1} \tilde{p}_{2}$, and yields a wave vector

$$
k_{\mathrm{bm}}= \pm \sqrt{-\frac{2 i \rho \omega w_{\mathrm{bm}}}{3 Z_{\mathrm{bm}}}\left(\frac{1}{A_{1}}+\frac{1}{A_{2}}\right)} .
$$

This wave vector does not involve deformation of the cochlear bone. Instead, it follows from the basilar-membrane impedance $Z_{\mathrm{bm}}$ alone and yields the well-known basilar-membrane wave.

As the basilar-membrane impedance varies longitudinally, the wave's amplitude changes as well. A Wentzel-Kramers-Brillouin (WKB) approximation can be applied and reveals that the local wave vector still follows from Equation (11), whereas the pressure amplitude is proportional to the inverse square root of the wave vector (Methods).

Second, and most important for our study here, the other eigenvector, $\tilde{p}_{2}=\tilde{p}_{1}$, involves pressures in both chambers that are equal at any given longitudinal location. The corresponding wave accordingly does not deflect the basilar membrane. It solely evokes deformation of the cochlear bone that propagates at a wave vector $k_{\mathrm{cb}}$ :

$$
k_{\mathrm{cb}}= \pm \sqrt{\frac{2 \rho \omega^{2} C}{A_{1}+A_{2}}} .
$$

We refer to this mode as the cochlear-bone wave. As the impedance of the cochlear bone remains approximately constant between the cochlear base and apex, this wave's amplitude remains constant as well and we do not need to employ the WKB approximation. The wavelength is the longer the larger the 
impedance of the cochlear bone. As this impedance is relatively high it yields a comparatively long wavelength, on the order of a few millimetres to a few centimetres, and accordingly a propagation speed that exceeds that of the basilar-membrane wave. Notably, the wavelength of the cochlear-bone wave is still substantially below that of a compressive fluid wave, which reflects the above finding that the fluid compressibility plays a negligible role.

Although the basilar-membrane and the cochlear-bone wave are clearly distinct, with one wave depending only on the basilarmembrane impedance and the other wave solely on the impedance of the cochlear bone, they couple in two intriguing ways. One type of coupling becomes important for otoacoustic emissions and the other for bone conduction.

As the first type of coupling, a force that acts on the basilar membrane can elicit the cochlear-bone wave. This unexpected effect becomes clear when we recall that a displacement of the basilar membrane increases the pressure in one chamber but decreases it in the other by the same amount, $\tilde{p}_{1}=-\tilde{p}_{2}$. Such displacement can elicit a wave on the basilar membrane that involves opposite pressures, $A_{2} \tilde{p}_{1}=-A_{1} \tilde{p}_{2}$. In an asymmetric cochlea, with $A_{1} \neq A_{2}$, the pressure changes evoked by basilarmembrane motion do not fully match those involved in the basilar-membrane wave. A force that acts on the basilar membrane must hence, besides the basilar-membrane wave, stimulate a second degree of freedom: the wave on the cochlear bone. As otoacoustic emissions arise from the activity of hair cells on the basilar membrane, they can hence excite a cochlear-bone wave and thus propagate out of the cochlea. Below we show this mechanism in detail for the case of distortion-product otoacoustic emissions.

In the second, in a sense reverse way of coupling, stimulation of the cochlear bone can elicit a basilar-membrane wave. Assume that, at a certain longitudinal location, both cochlear chambers change their area by the same amount due to forcing. As the cross-sectional areas of both chambers are, in general, different, such forcing produces different pressures in the two chambers and hence a displacement of the basilar membrane. This mechanism can yield bone conduction as we show below.

Distortion products. Distortion products are combination tones that the cochlea produces when it encounters multiple frequencies. As a prominent example, when stimulated by two close frequencies $f_{1}$ and $f_{2}$, in which $f_{1}$ is smaller than $f_{2}$ by convention, the inner ear yields emissions at cubic distortion frequencies such as $2 f_{1}-f_{2}$ and $2 f_{2}-f_{1}$.

This distortion is produced by a nonlinearity on the basilar membrane. Indeed, close to its resonant position, the linear response (equation (6)) of the basilar membrane is supplemented by a cubic nonlinearity that originates in the amplification provided by hair cells:

$$
\tilde{p}_{1}-\tilde{p}_{2}=Z_{\mathrm{bm}} \tilde{V}_{\mathrm{bm}}+3 A \widetilde{V_{\mathrm{bm}}^{3}}
$$

in which $A$ is a coefficient. Distortion arising for the Fourier transform of the cubic nonlinearity can be written as the convolution of Fourier coefficients: $\widetilde{V_{\mathrm{bm}}^{3}}=\tilde{V}_{\mathrm{bm}} * \tilde{V}_{\mathrm{bm}} * \tilde{V}_{\mathrm{bm}}$, which yields mixing in the frequency domain.

To solve the nonlinear equation (13), we first compute Green's functions, that is, pressures $\tilde{p}_{1 / 2}^{G}\left(x, x_{0}, \omega\right)$ that result from a single force at position $x_{0}$ :

$$
\tilde{p}_{1}^{G}\left(x, x_{0}, \omega\right)-\tilde{p}_{2}^{G}\left(x, x_{0}, \omega\right)=Z_{\mathrm{bm}} \tilde{V}_{\mathrm{bm}}+p_{F} \cos (\omega t) \delta\left(x-x_{0}\right) .
$$

Using techniques from complex analysis, we obtain an analytical solution for these Green's functions (Methods). The solution consists of two waves modes, the basilar-membrane wave as well as the wave on the cochlear bone. The latter is excited
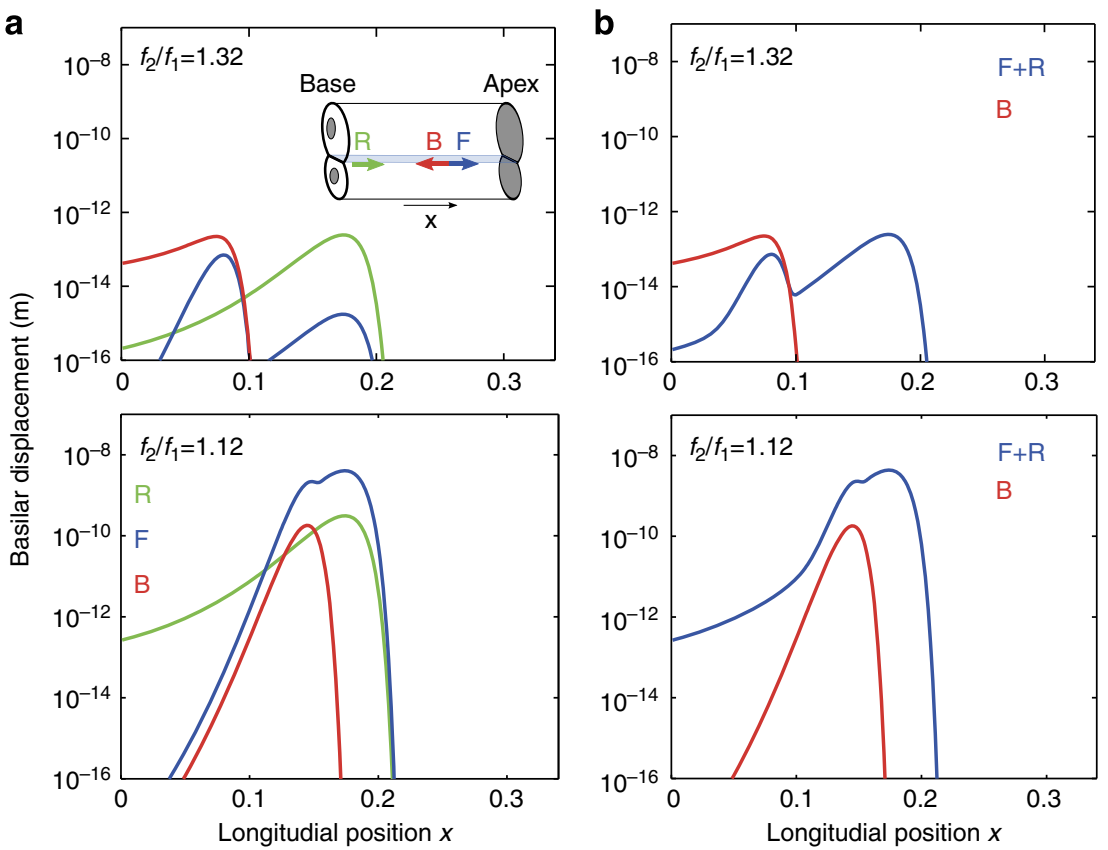

Figure 3 | Distortion-product waves travelling towards and away from the base. (a) Amplitude of basilar-membrane waves at distortion frequency $f=2 f_{1}-f_{2}=7 \mathrm{kHz}$. One component travels backward from the generation site toward the base (' $B$ ', red), whereas another propagates forward to the apex (' $F$ ', blue). A third wave emerges from the reflection of the backward-propagating cochlear-bone wave and travels forward on the membrane (' $R$ ', green). The three contributions are illustrated in the inset. The upper and lower panel show the amplitudes for two different ratios of the primary frequencies. (b) The reflected and the forward-travelling basilar-membrane wave combine to a net forward-travelling wave (' $F+R^{\prime}$, blue). Depending on the ratio of the primary frequencies as well as the cochlear location, this wave can overwhelm the backward-propagating one (' $B$ ', red). 
when the cochlear chambers are asymmetric, $A_{1} \neq A_{2}$. In this case, the nonlinear basilar-membrane response accordingly produces not only a basilar-membrane wave but also a cochlear-bone wave.

Within each wave mode, two distinct waves emerge. First, one wave travels backward from the generation site $x_{0}$ to the stapes. The second wave moves forward to the apex. Although it may undergo reflection at the apex, we ignore this forward-travelling wave in the following and only consider the wave that travels backward.

As the cochlear nonlinearity extends over a certain region near the peaks of the primary frequencies, many such waves are produced and add up to yield the net distortion product. Mathematically, this follows from integrating the Green's functions (equation (14)) together with the nonlinear inhomogeneity $\widetilde{V_{\mathrm{bm}}^{3}}$, which yields the solution to the inhomogeneous differential equation (13):

$$
\tilde{p}_{1 / 2}(x, \omega)=\frac{3 A}{p_{F}} \int_{0}^{L} d x_{0} \tilde{p}_{1 / 2}^{G}\left(x, x_{0}, \omega\right) \widetilde{V_{\mathrm{bm}}^{3}}\left(x_{0}, \omega\right) .
$$

What happens to the backward-travelling waves, the one in the basilar-membrane and the other in the cochlear-bone mode? Part of the energy that they carry will be emitted into the ear canal. The remainder will be reflected off the middle ear and produce forward-travelling waves. One such wave will propagate on the basilar membrane, and the other as cochlear-bone deformation.

The reflection of the backward-propagating waves off the middle ear can be quantified by considering the action of the middle ear (Methods). Indeed, the middle ear acts as an impedance transformer that matches the impedance of an incoming sound to that of the basilar-membrane wave. An incoming sound is hence largely transmitted to basilar-membrane motion, without much reflection at the middle ear. Reversely, a backward-propagating basilar-membrane wave is effectively transmitted to a sound wave, and not much reflection occurs. A backward-propagating cochlear-bone wave, in contrast, will be much less transmitted because its impedance differs from the basilar-membrane wave and is not matched by the middle ear. Considerable reflection then occurs and produces forwardtravelling waves, in particular, a wave on the basilar membrane.

Three basilar-membrane waves hence propagate at the distortion frequency (Fig. 3a). First, a forward-travelling wave is generated by the basilar-membrane's nonlinearity. This wave is predominantly created in the region where the primary frequencies overlap. As the contributions from this region differ in phase, they partly cancel, and the wave has an amplitude peak at the point of maximal generation. For the lower sideband distortion frequency $2 f_{1}-f_{2}$ that we consider here, the wave then travels further apical and experiences a second peak near its resonant position.

Second, the nonlinear basilar-membrane response creates a backward-propagating wave as well. As for the forward-travelling wave, the contributions to this wave from different cochlear locations partly annihilate each other, and the amplitude of this wave is largest at the point of maximal generation. The wave cannot be created apical to the resonant position of the upper primary frequency, $f_{2}$, such that no backward wave arises there.

Third, a reflected forward-travelling wave arises from the reflection of the reverse basilar-membrane and the cochlear-bone wave. This wave's amplitude behaves as the usual basilarmembrane wave: its amplitude increases until it reaches its resonant position, beyond which it sharply diminishes.

The first and third component superimpose to yield the net forward-travelling wave on the basilar membrane. Can that wave have a larger amplitude than the reverse basilar-membrane wave and hence conceal its existence?.

Our numerical simulations show that the answer depends on the ratio of the primary frequencies as well as, potentially, on the cochlear location (Fig. 3b). When the primary frequencies are sufficiently apart, the reverse wave can blanket the forwardpropagating waves. Close primary frequencies, however, yield a net forward-travelling wave that exceeds the backwardpropagating one at all cochlear locations.

In order to intuitively understand these results, we recall that the distortion is generated within an extended cochlear region, namely where the peaks of the primary-frequency waves significantly overlap. The phase of the distortion changes with location, and the produced reverse-propagating waves hence experience significant destructive interference. This destructive interference is the stronger the faster the phase changes, and hence the smaller the wavelength is. Generation close to the peak region, where the basilar-membrane wavelength is short, yields accordingly more destructive interference. Similarly, because the cochlear-bone wave has a comparably long wavelength, its generation comes with less destructive interference than that of the basilar-membrane wave.

As the basilar-membrane waves of closer primary frequencies overlap stronger, they produce more destructive interference in the generated, reverse basilar-membrane wave. In relation to the latter the produced backward-travelling cochlear-bone wave is therefore stronger and yields accordingly a stronger reflection. Part of that
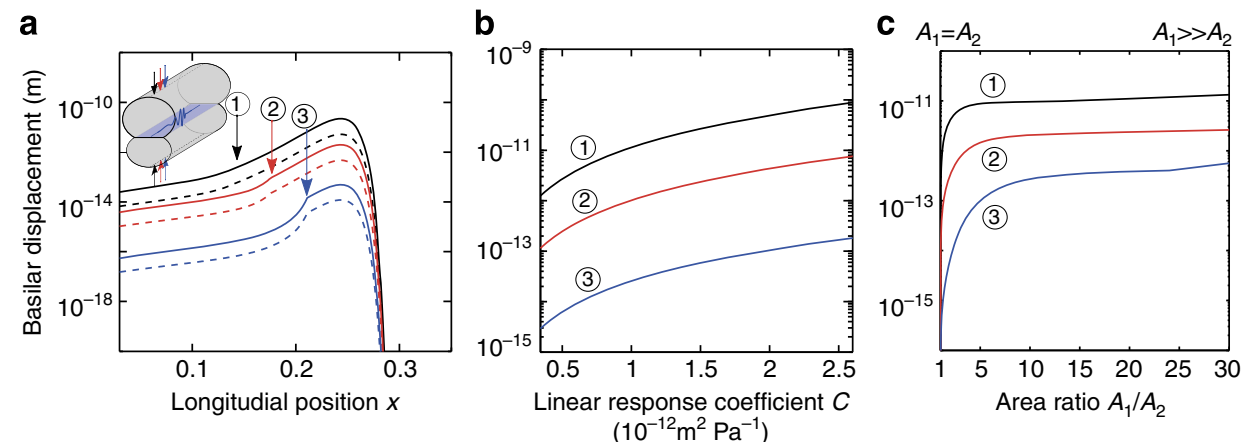

Figure 4 | Basilar-membrane excitation through cochlear-bone compression. (a) Deformation of the cochlear chambers at different location $x$, illustrated by the numbered vertical arrows, can deflect the basilar membrane. The inset schematically shows the compressive stimulation of the cochlear bone; cochlear bone linear response $C=6.86 .10^{-13} \mathrm{~m}^{2} \mathrm{~Pa}^{-1}$. (b) The maximal basilar-membrane deflection depends on the material properties of the cochlear bone. A larger linear-response coefficient of the bone implies a smaller bone impedance, which then is closer to that of the basilar membrane and yields a greater basilar-membrane deflection. (c) The maximal basilar-membrane deflection depends on the ratio $A_{1} / A_{2}$ of the cross-sectional areas $A_{1}$ and $A_{2}$ of the two chambers. The membrane displacement vanishes in a symmetric cochlea $\left(A_{1} / A_{2}=1\right)$ and grows with increasing asymmetry $\left(A_{1} \ll A_{2}\right)$. 
reflection is a forward-travelling basilar-membrane wave that hence blankets the reverse wave on the basilar membrane.

Bone conduction. Deformation of the cochlear bone can elicit basilar-membrane waves and hence a hearing sensation. Similar to our calculations regarding distortion-product otoacoustic emissions, we quantify this effect through computing Green's functions, that is, the pressure waves that result from deforming the cochlear bone at a single longitudinal location $x_{0}$ (Methods). Specifically, we consider a deformation of the cochlear bone such that the cross-sectional area of the upper chamber vibrates in phase with that of the lower chamber, and with the same amplitude.

The Green's functions show that four waves emerge from such stimulations: two cochlear-bone waves, travelling basally and apically from the stimulation site, and two basilar-membrane waves, also propagating backward and forward. The basilarmembrane waves are hereby only excited if the two chambers differ in their cross-sectional area, $A_{1} \neq A_{2}$. In a hypothetical symmetric inner ear, in which the areas are equal, deformation of the cochlear bone would not elicit basilar-membrane waves, as had already been remarked by Békésy ${ }^{24}$.

We are interested in the basilar-membrane waves because they elicit the hearing sensation. Apical to the stimulation point, we find a forward-travelling wave that peaks close to its resonant position and resembles the standard, middle-ear-evoked waves for all stimulation points (Fig. 4a). Basal to the stimulation point we obtain a backward-travelling wave that decays in amplitude as it travels towards the base. The amplitude of the elicited basilarmembrane wave depends on the stimulation position along the cochlea: it increases for more basal stimulation. The shape of the produced wave is, however, largely independent of the location of stimulation. Compressive stimulation of an extended region of the cochlear bone generates a superposition of the waves elicited by point stimulation. The extent of the stimulation region governs the amplitude but not the spatial profile of the basilar-membrane motion.

The amplitude of the elicited basilar-membrane motion depends on the impedance of the cochlear bone as compared with the membrane's (Fig. 4b). The impedance associated to bone deformation is generally higher than that of the basilar membrane. The smaller the bone's impedance, the more similar it becomes to that of the membrane. Deformation of the cochlear bone then couples stronger to the basilar-membrane wave and produces a larger amplitude.

The asymmetry between the two cochlear chambers, measured through the ratio $A_{1} / A_{2}$ of their cross-sectional areas, is another important factor in this mechanism as stated above (Fig. 4c). In a symmetric cochlea, deformation of the cochlear bone does not produce a deflection of the basilar membrane. In a real cochlea, however, the cross-sectional areas of both chambers differ. The evoked basilar-membrane vibration is the stronger the larger the asymmetry is.

\section{Discussion}

Our results show that deformation of the cochlear bone can play a critical role in sound perception as well as in the propagation of otoacoustic emissions. Deformation of the cochlear bone can yield a fast wave, in addition to the much-studied slow basilarmembrane wave. As the cochlea is asymmetric-the crosssectional areas of both chambers differ-the two modes couple to each other.

A force that acts on the basilar membrane, such as the one produced by the activity of hair cells, elicits not only a wave on the membrane but a wave on the cochlear bone as well. We have shown how distortion on the basilar membrane can accordingly produce an otoacoustic emission that emerges from the inner ear through propagating from its generation site back to the stapes as cochlear-bone deformation. As the wavelength of the cochearbone mode is relatively long, of the order of a centimetre and hence comparable to the dimensions of the inner ear, the temporal delay of this emission is small: the backwardpropagating wave reaches the middle ear quickly. This mechanism can hence underlie the short-delay component of an otoacoustic emission.

Previously, it has been suggested that the nonlinear distortion produced by basilar-membrane vibration can launch a compressive fluid wave that propagates back to the stapes ${ }^{19-21}$. Our computations show that, when the cochlear bone is deformable, this wave involves not only the compression of the fluid but also deformation of the cochlear chambers. In fact, the latter effect dominates because the impedance associated to deformation of the cochlear bone is much less than that associated to compression of the fluid (equation (10)). The wave accordingly has a significantly shorter wavelength than an ordinary compressive fluid wave.

The distortion in the cochlea also produces a reverse wave on the basilar membrane. Why has this component not been detected in recent laser-interferometric experiments?

Our modelling reveals that a sizable portion of the backwardtravelling wave on the cochlear bone becomes reflected at the middle ear and propagates forward to the cochlear apex, both as a wave on the basilar membrane and as a cochlear-bone wave. We have quantified the magnitude of the reflected, forward-travelling basilar-membrane wave. As close primary frequencies are typically used in experiments, the forward wave can have a significantly higher amplitude than the reverse basilar-membrane wave. Experiments will then only detect the forward-travelling wave. The stapes will accordingly vibrate before the basilar membrane, because the main component of basilar-membrane vibration arises from reflection at the stapes and hence occurs at a certain temporal delay. This delay has been measured in recent experiments ${ }^{19}$.

Our study shows that the backward-propagating basilarmembrane wave may dominate when the primary frequencies are sufficiently far apart. It will be interesting to see whether this reverse wave can indeed be experimentally measured or whether its amplitude is too tiny, because distortion at far primary frequencies is small.

The one-dimensional model that we have employed cannot account for the drop in pressure near the peak of the basilar-membrane wave when deviating vertically from the membrane. This pressure drop may alter the coupling to the cochlear-bone wave, which may be interesting for future studies.

Stimulation of the cochlear bone-as elicited by boneconduction headphones, for instance-can produce a basilarmembrane wave and accordingly yield a hearing sensation. We have calculated the vibration of the basilar membrane and how it varies longitudinally. Our results show a basilar-membrane wave that closely resembles the wave that emerges from airborne sound. The amplitude is the stronger the larger the difference in the cross-sectional areas of the two cochlear chambers. It also depends on the material properties of the cochlear bone. For realistic parameter values the amplitude of the membrane vibration corresponds to the experimentally-observed magnitude of bone conduction.

The increasing development and usage of bone-conduction headphones such as in the Google glass device and other commercial applications points to a need for a conceptual understanding of the underlying biophysics. We hope that the 
results we presented here help to clarify the mechanisms involved in bone conduction, and to further advance its application.

\section{Methods}

Parabolic deflection of the basilar membrane. We assume that each transverse segment of the basilar membrane deflects parabolically. The membrane's width is $w_{\mathrm{bm}}$, and we choose a transverse coordinate $y$ such that $y=-w_{\mathrm{bm}} / 2$ and $y=w_{\mathrm{bm}} / 2$ denote the points where the membrane segment is anchored in bone. The membrane velocity $V(y, t)$ is then

$$
V(y, t)=\frac{4 V_{\mathrm{bm}}}{w_{\mathrm{bm}}^{2}}\left(y-\frac{w_{\mathrm{bm}}}{2}\right)\left(y+\frac{w_{\mathrm{bm}}}{2}\right),
$$

in which $V_{\mathrm{bm}}$ is the maximal basilar-membrane velocity (at its midpoint $y=0$ ).

The temporal changes $\partial_{t} a_{1}$ and $\partial_{t} a_{2}$ of the cochlear chambers' cross-sectional areas are then as follows:

$$
\partial_{t} a_{1}=-\partial_{t} a_{2}=\int_{-\frac{m_{\mathrm{bm}}}{2}}^{\frac{m_{\mathrm{bm}}}{2}} \mathrm{~d} y V(y, t)
$$

which yields equation (3).

Linear-response coefficient $\boldsymbol{C}$. We consider a tube subject to radial pressure The tube's wall is assumed to be incompressible and elastic such that the circumference of a cross-section of the tube remains constant under deformation.

We assume that the cross-section of the tube is approximately elliptical, with a wall distance $r_{0}$ from the midpoint that depends on the central angle $\phi$ through $r_{0}(\phi)=R+w_{0} \cos (2 \phi)$ (ref. 26). The variable $w_{0}$ hence measures the deviation of the cross-sectional shape from a circle, and the variable $R$ denotes the average wall distance.

A change $p$ in the internal radial pressure leads to a deformation $r(\phi)$ that we describe through a variable $w: r(\phi)=R+w \cos (2 \phi)$. The magnitude of the change $\delta w=w-w_{0}$ is derived in equations (7-18) in the study by Timoshenko and Gere ${ }^{26}$.

$$
\delta w=-\frac{w_{0} p}{p_{c r}-p}, \text { with } p_{c r}=\frac{E}{4\left(1-v^{2}\right)} \frac{h^{3}}{R^{3}} .
$$

Here, $E$ denotes the Young's modulus of the cochlear bone, $v$ the Poisson ratio and $h$ the thickness of the cochlear bone.

A small pressure change $p$ elicits an approximately proportional change $\delta w$ :

$$
\delta w \approx \frac{4\left(1-v^{2}\right)}{E} \frac{R^{3} w_{0}}{h^{3}} p .
$$

A small change $\delta w$ in the variable $w$ leads, in turn, to a small area change. The area $A_{\mathrm{cs}}$ of a cross-section can be computed from $r(\phi)$ as

$$
A_{\mathrm{cs}}=\frac{1}{2} \int_{0}^{2 \pi} r^{2} \mathrm{~d} \phi
$$

The area change $a$ follows, to first order in the change $\delta w$, as

$$
a=\frac{\partial A_{\mathrm{cs}}}{\partial w} \delta w=\frac{1}{2} \int_{0}^{2 \pi} 2 r \frac{\partial r}{\partial w} \delta w \mathrm{~d} \phi=\pi w_{0} \delta w .
$$

The small pressure change $p$ hence induces an area change according to $a=C p$, with the coefficient

$$
C=\frac{4 \pi\left(1-v^{2}\right)}{E} \frac{R^{3} w_{0}^{2}}{h^{3}}
$$

The latter is the linear-response coefficient that we employ in Equation (7).

Spatial impedance variation and WKB approximation. The impedance of the basilar membrane varies systematically along the cochlea. The basilar-membrane wave accordingly changes its wavelength as it propagates from the base towards its resonant position. The change of the wavelength and the amplitude can be captured by the WKB approximation, which starts from the following ansatz for the pressures $^{2}$ :

$$
p_{1 / 2}=\tilde{p}_{1 / 2}(x) e^{i \omega t-\Phi_{1 / 2}(x)}+\text { c.c. }
$$

To fulfil the wave equation the amplitudes $\tilde{p}_{1 / 2}(x)$ and phases $\Phi_{1 / 2}(x)$ have to obey

$$
\begin{aligned}
& \partial_{x}^{2} \tilde{p}_{1 / 2}(x)+2 i \partial_{x} \tilde{p}_{1 / 2}(x) \partial_{x} \Phi_{1 / 2}(x)-\tilde{p}_{1 / 2}(x)\left[\partial_{x} \Phi_{1 / 2}(x)\right]^{2}+i \tilde{p}_{1 / 2}(x) \partial_{x}^{2} \Phi_{1 / 2}(x) \\
& \quad=-\tilde{p}_{1 / 2}(x) \kappa(x)^{2} .
\end{aligned}
$$

The real part $\partial_{x}^{2} \tilde{p}_{1 / 2}(x)+\tilde{p}_{1 / 2}(x)\left\{\kappa(x)^{2}-\left[\partial_{x} \Phi_{1 / 2}(x)\right]^{2}\right\}=0$ implies that $\Phi_{1 / 2}(x)= \pm \int_{0}^{x} \kappa\left(x^{\prime}\right) \mathrm{d} x^{\prime}$. The imaginary part, $2 \partial_{x} \tilde{p}_{1 / 2}(x) \partial_{x} \Phi(x)$ $+\tilde{p}_{1 / 2}(x) \partial_{x}^{2} \Phi(x)=0$, leads to $\tilde{p}_{1 / 2}(x)=\phi_{i} / \sqrt{\kappa(x)}$.
Green's functions. Green's functions are pressures that result from point-wise stimulation at $x_{0}$ along the cochlea at frequency $\omega$. Two types of Green's functions are important in our study. The first type, pressures $\tilde{p}_{1 / 2}^{G}\left(x \mid x_{0}, \omega\right)$, reflects stimulation of the basilar membrane. The second type, pressures $\tilde{p}_{1 / 2}^{W}\left(x \mid x_{0}, \omega\right)$, arise from stimulating the cochlear bone.

We start with computing the Green's functions that result from a point force acting on the basilar membrane. Such a force appears in the the boundary condition (equation (14)). We make the ansatz

$$
\begin{aligned}
& \tilde{p}_{1}^{G}\left(x \mid x_{0}, \omega\right)=\int_{-\infty}^{\infty} \mathrm{d} k G_{1}(\kappa) e^{i \omega t-i k\left(x-x_{0}\right)}, \\
& \tilde{p}_{2}^{G}\left(x \mid x_{0}, \omega\right)=\int_{-\infty}^{\infty} \mathrm{d} k G_{2}(\kappa) e^{i \omega t-i k\left(x-x_{0}\right),}
\end{aligned}
$$

with the wave-vector-dependent coefficients $G_{1}(k)$ and $G_{2}(k)$. Using fluidmomentum equation (1) with the continuity equation (2) as well as equations (7) and (14)) we obtain two coupled ordinary differential equations,

$$
\begin{gathered}
-i \omega \rho\left\{\frac{2 \omega_{\mathrm{bm}}}{3 Z_{\mathrm{bm}}(x)}\left[G_{1}(\kappa)-G_{2}(\kappa)-\frac{p_{F}}{2 \pi}\right]+i \omega c G_{1}(\kappa)\right\}=A_{1} k^{2} G_{1}(\kappa), \\
i \omega \rho\left\{\frac{2 \omega_{\mathrm{bm}}}{3 Z_{\mathrm{bm}}(x)}\left[G_{1}(\kappa)-G_{2}(\kappa)-\frac{p_{F}}{2 \pi}\right]-i \omega c G_{2}(\kappa)\right\}=A_{2} k^{2} G_{2}(\kappa) .
\end{gathered}
$$

The coefficients $G_{1}(k)$ and $G_{2}(k)$ are defined as follows:

$$
\begin{gathered}
G_{1}(\kappa)=\frac{i \omega p_{F} \rho\left(A_{2} k^{2}-c \omega^{2} \rho\right) w_{\mathrm{bm}}}{3 A_{1} A_{2} \pi Z_{\mathrm{bm}}(x) L(\kappa)}, \\
G_{2}(\kappa)=\frac{i \omega p_{F} \rho\left(-A_{1} \kappa^{2}+c \omega^{2} \rho\right) w_{\mathrm{bm}}}{3 A_{1} A_{2} \pi Z_{\mathrm{bm}}(x) L(\kappa)} .
\end{gathered}
$$

Here we have used the abbreviation $L(k)=\left[2 i \omega \rho\left(F_{1}+F_{2}\right) w_{\mathrm{bm}}+3 F_{1} F_{2} Z_{\mathrm{bm}}(x)\right] /$ $\left[3 A_{1} A_{2} Z_{\mathrm{bm}}(x)\right]$ with $F_{1 / 2}=A_{1 / 2} k^{2}-c \omega^{2} \rho$. The dispersion relation $L(k)=0$ has been derived from the eigenvalues of the matrix $\mathcal{M}$ equation (10).

The Green's functions for bone stimulation can be derived analogously. Assume that both cochlear chambers, at a certain longitudinal location $x_{0}$, are sinusoidally compressed and expanded:

$$
\widetilde{a_{1 / 2}}=\frac{2 i}{3 \omega} w_{\mathrm{bm}} \tilde{V}_{\mathrm{bm}}+C\left[\tilde{p}_{1 / 2}+p_{F} \cos (\omega t) \delta\left(x-x_{0}\right)\right]
$$

We make the following ansatz for the Greens functions:

$$
\begin{aligned}
& \tilde{p}_{1}^{W}\left(x \mid x_{0}, \omega\right)=\int_{-\infty}^{\infty} d \kappa W_{1}(\kappa) e^{i \omega t-i \kappa\left(x-x_{0}\right)}, \\
& \tilde{p}_{2}^{W}\left(x \mid x_{0}, \omega\right)=\int_{-\infty}^{\infty} d \kappa W_{2}(\kappa) e^{i \omega t-i \kappa\left(x-x_{0}\right)},
\end{aligned}
$$

which yields the amplitude equations

$$
\begin{gathered}
-i \omega \rho\left[\frac{2 \omega_{\mathrm{bm}}}{3 Z_{\mathrm{bm}}}\left(p_{1}^{W}-p_{2}^{W}\right)+i c \omega\left(p_{1}+\frac{p F}{2 \pi}\right)\right]=A_{1} \kappa^{2} p_{1} \\
i \omega \rho\left[\frac{2 \omega_{\mathrm{bm}}}{3 Z_{\mathrm{bm}}}\left(p_{1}^{W}-p_{2}^{W}\right)-c i \omega\left(p_{2}+\frac{p F}{2 \pi}\right)\right]=A_{2} \kappa^{2} p_{2} .
\end{gathered}
$$

The solutions are

$$
\begin{aligned}
& W_{1}(\kappa)=\frac{c \omega^{2} p_{F} \rho\left[4 i \omega \rho w_{\mathrm{bm}}+3 A_{2} \kappa^{2} Z_{\mathrm{bm}}(x)-3 c \omega^{2} \rho Z_{\mathrm{bm}}(x)\right]}{6 A_{1} A_{2} \pi Z_{\mathrm{bm}}(x) L(\kappa)}, \\
& W_{2}(\kappa)=\frac{c \omega^{2} p_{F} \rho\left[4 i \omega \rho w_{\mathrm{bm}}+3 A_{1} k^{2} Z_{\mathrm{bm}}(x)-3 c \omega^{2} \rho Z_{\mathrm{bm}}(x)\right]}{6 A_{1} A_{2} \pi Z_{\mathrm{bm}}(x) L(\kappa)} .
\end{aligned}
$$

with $L(k)$ as given above. In the symmetric case of equal chamber areas, $A_{1}=A_{2}$, we obtain $W_{1}(k)=W_{2}(k)$. No basilar-membrane displacement then arises, because the pressures in both chambers are equal.

When attempting to compute the integral in the ansatz for the Green's functions (equations (25) and (29)), we encounter a problem: the integrand has a singularity at the wave vectors $k$ for which $L(k)=0$, that is, at those wave vectors that obey the dispersion relation. However, we can employ the residue theorem of complex analysis to compute the integrals. Indeed, for propagation apical of the generation site, that is at a location $x<x_{0}$, we can close the contour in the upperhalf plane because the integrand there is exponentially suppressed. The integral then only involves a contribution from the poles in the upper-half plane. In the case of basilar-membrane stimulation, we obtain a contribution proportional to $\left[\partial_{k} W_{1 / 2}^{-1}(\kappa)\right]^{-1}$. The pressures $p_{1 / 2}\left(-k_{\mathrm{bm}}, \omega, x_{0}\right)$ represent the pressures of the basilar membrane mode in the two chambers

$$
\begin{aligned}
& p_{1}\left(-\kappa_{\mathrm{bm}}, \omega, x_{0}\right)=\left.2 \pi i \frac{\sqrt{\kappa_{\mathrm{bm}}\left(x_{0}\right)}}{\sqrt{\kappa_{\mathrm{m}}(x)}}\left(\frac{\partial}{\partial k} W_{1}(\kappa)^{-1}\right)^{-1}\right|_{\kappa=-\kappa_{\mathrm{bm}}\left(x_{0}\right)} \cdot e^{i \int_{x}^{x_{0}} \kappa_{\mathrm{bm}}(x) d x+i \omega t}+c . c ., \\
& p_{2}\left(-\kappa_{\mathrm{bm}}, \omega, x_{0}\right)=\left.2 \pi i \frac{\sqrt{\kappa_{\mathrm{m}}\left(x_{0}\right)}}{\sqrt{\kappa_{\mathrm{bm}}(x)}}\left(\frac{\partial}{\partial k} W_{2}(\kappa)^{-1}\right)^{-1}\right|_{k=-\kappa_{\mathrm{bm}}\left(x_{0}\right)} \cdot e^{i \int_{x}^{x_{0}} \kappa_{\mathrm{bm}}(x) d x+i \omega t}+c . c .
\end{aligned}
$$

Analogous results can be obtained for the cochlear-bone wave with $k_{\mathrm{cb}}(x)$

In the opposite case, for a cochlear location basal to the generation site, $x>x_{0}$, the integration path can be closed in the lower-half plane.

Middle ear pressure transformation. The three ossicles of the middle ear-malleus, incus and stapes-connect the ear drum to the oval window. Sound is accordingly transmitted from the ear canal to the cochlea, and can analogously be re-emitted from the cochlea into the ear canal. How can these transfers be quantified?

Denote by $A_{\mathrm{a}}$ and $A_{\mathrm{ow}}$ the area of the tympanic membrane with respect to the oval window, and by $l_{\mathrm{a}}$ and $l_{\mathrm{w}}$ the length of the mallus with respect to the incus (Fig. 1). The pressure in the ear canal is $p_{3}$, it acts on the tympanic membrane and produces an angular momentum $l_{\mathrm{a}} A_{\mathrm{a}} p_{3}$. The pressure $p_{2}$ in the upper cochlear 
chamber yields an angular momentum $l_{\mathrm{w}} A_{\mathrm{ow}} p_{2}$, which must match the first one:

$$
l_{\mathrm{a}} A_{\mathrm{a}} p_{3}=l_{\mathrm{w}} A_{\text {ow }} p_{2} \text {. }
$$

A second equation results from the fluid flows in the ear canal as well as in the upper cochlear chamber, $j_{3}$ and $j_{2}$, which must yield an equal angular deflection of the middle-ear bones:

$$
\frac{j_{3}}{l_{\mathrm{a}} A_{\mathrm{a}}}=\frac{j_{2}}{l_{\mathrm{w}} A_{\mathrm{ow}}} .
$$

Finally, the pressure $p_{1}$ in the lower cochlear chamber creates a fluid flow $j_{1}$ at the round window that depends on its impedance $Z_{\mathrm{rw}}{ }^{30}$ :

$$
p_{1}=Z_{\mathrm{rw}} j_{1} \text {. }
$$

These three equations act as boundary conditions to the wave equations and allow to compute the extent to which a wave reaching the middle ear, either from the ear canal or from within the cochlea, is transmitted or reflected.

We first illustrate how this computation works by considering airborne sound travelling through the ear canal towards the tympanic membrane, with a wave vector $\kappa_{s}=\omega \sqrt{\rho_{\text {air }} \kappa}$ in which $\rho_{\text {air }}$ and $\kappa$ are the air's density with respect to compressibility. Part of this wave will be reflected, such that the pressure in the ear canal is the sum of a forward- and a backward-travelling sound wave:

$$
p_{3}=\tilde{p}_{3, f} e^{i \omega t-i k_{s} x}+\tilde{p}_{3, b} e^{i \omega t+i k_{s} x} .
$$

Within the cochlea, forward-travelling waves on the basilar membrane (wave vector $k_{\mathrm{bm}}$ ) as well as on the cochlear bone (wave vector $k_{\mathrm{cb}}$ ) will be elicited:

$$
p_{1 / 2}=\tilde{p}_{1 / 2, \mathrm{bm}} e^{i \omega t-i k_{\mathrm{bm}} x}+\tilde{p}_{1 / 2, \mathrm{cb}} e^{i \omega t-i k_{\mathrm{cb}} x} .
$$

The associated fluid flows at the middle ear can be obtained from equation (1) in which the cross-sectional areas are substituted by the corresponding membrane areas, namely the ones of the tympanic membrane, round and oval window. The boundary equations (33-35) can then be solved for the amplitudes of the wave components:

$$
\begin{aligned}
& \tilde{p}_{3, b}=\tilde{p}_{3, f} \frac{\left[A_{\mathrm{ow}} k l_{\mathrm{w}}^{2} \rho\left(H \omega \rho-A_{\mathrm{rw}} K_{2} Z_{\mathrm{rw}}\right)+A_{\mathrm{a}} l_{\mathrm{a}}^{2} \rho_{\mathrm{air}}\left(-K_{1} \omega \rho+A_{\mathrm{rw}} H k_{\mathrm{bm}} k_{\mathrm{cb}} Z_{\mathrm{rw}}\right)\right]}{A_{\mathrm{ow}} l_{\mathrm{w}}^{2} \rho\left(H \omega \rho-A_{\mathrm{rw}} K_{2} Z_{\mathrm{rw}}\right)+A_{\mathrm{a}} l_{a}^{2} a_{\mathrm{air}}\left(K_{1} \omega \rho-A_{\mathrm{rw}} H k_{\mathrm{bm}} k_{\mathrm{cb}} Z_{\mathrm{rw}}\right)}, \\
& \tilde{p}_{2, \mathrm{bm}}=\tilde{p}_{3, f} \frac{2 A_{\mathrm{a}} A_{1} k_{l_{1}} l_{\mathrm{w}} \rho\left(\omega \rho-A_{\mathrm{rw}} k_{\mathrm{cb}} Z_{\mathrm{rw}}\right)}{A_{\mathrm{ow}} k l_{\mathrm{ww}}^{2} \rho\left(H \omega \rho-A_{\mathrm{rw}} K_{2} Z_{\mathrm{rw}}\right)+A_{\mathrm{a}} l_{\mathrm{a}}^{2} \rho_{\mathrm{air}}\left(K_{1} \omega \rho-A_{\mathrm{rw}} H k_{\mathrm{bm}} k_{\mathrm{cb}} Z_{\mathrm{rw}}\right)}, \\
& \tilde{p}_{2, \mathrm{cb}}=\tilde{p}_{3, f} \frac{2 A_{\mathrm{a}} A_{2} k l_{\mathrm{a}} l_{\mathrm{w}} \rho\left(\omega \rho-A_{\mathrm{rw}} k_{\mathrm{bm}} Z_{\mathrm{rw}}\right)}{A_{\mathrm{ow}} k l_{\mathrm{w}}^{2} \rho\left(H \omega \rho-A_{\mathrm{rw}} K_{2} Z_{\mathrm{rw}}\right)+A_{\mathrm{a}} l_{\mathrm{a}} \rho_{\mathrm{air}}\left(K_{1} \omega \rho-A_{\mathrm{rw}} H k_{\mathrm{bm}} k_{\mathrm{cb}} Z_{\mathrm{rw}}\right)} .
\end{aligned}
$$

Here we have employed the following abbreviations: $K_{1}=A_{1} k_{\mathrm{bm}}+A_{2} k_{\mathrm{cb}}$, $K_{2}=A_{2} k_{\mathrm{bm}}+A_{1} k_{\mathrm{cb}}, H=A_{1}+A_{2}$.

The middle ear matches impedances such that most of the energy of the sound wave is transmitted to the basilar-membrane wave. We employ this criterion to determine the impedance of the round window. Requiring that the incoming sound wave is not reflected at the middle ear but instead fully transmitted into the cochlea, we obtain the impedance of the round window as:

$$
Z_{\mathrm{rw}}=\frac{\omega \rho\left(A_{\mathrm{ow}} H k l_{\mathrm{w}}^{2} \rho-A_{\mathrm{a}} K_{1} l_{\mathrm{a}}^{2} \rho_{\text {air }}\right)}{A_{\mathrm{rw}}\left(A_{\mathrm{ow}} k K_{2} l_{\mathrm{w}}^{2} \rho-A_{\mathrm{a}} H k_{\mathrm{bm}} \kappa_{\mathrm{cb}} l_{\mathrm{a}}^{2} \rho_{\text {air }}\right)} .
$$

Next, we consider how a distortion signal emerges from the cochlea through a cochlear-bone wave. To this end we compute how much of a backward cochlearbone wave, as generated from distortion, is transmitted as a sound wave into the ear canal, and how much is reflected as forward-travelling wave in the cochlea (potentially both in the cochlear-bone and in the basilar-membrane mode). We hence start from the following ansatz

$$
p_{1 / 2}=\tilde{p}_{1 / 2, \mathrm{cb}, b} e^{i \omega t+i k_{\mathrm{cb}} x}+\tilde{p}_{1 / 2, \mathrm{cb}, f} e^{i \omega t-i k_{\mathrm{cb}} x}+\tilde{p}_{1 / 2, \mathrm{bm}} e^{i \omega t-i k_{\mathrm{bm}} x}, p_{3}=\tilde{p}_{3} e^{i \omega t+i k_{s} x},
$$

in which $\tilde{p}_{1 / 2, \mathrm{cb}, b}$ is the amplitude of the backward-propagating bone wave, $\tilde{p}_{1 / 2, \mathrm{cb}, f}$ the amplitude of the forward-travelling cochlear-bone wave, $\tilde{p}_{1 / 2, b m}$ the amplitude of the forward-propagating basilar-membrane wave and $\tilde{p}_{3}$ the amplitude of the emitted sound wave. From equations (1) and (33-35) we compute those amplitudes as:

$$
\begin{gathered}
\tilde{p}_{3}=\tilde{p}_{2, \mathrm{cb}, b} \frac{4 A_{\mathrm{a}} A_{\mathrm{ow}} H A_{2} \kappa \kappa_{\mathrm{cb}} l_{\mathrm{a}}^{2} l_{\mathrm{w}}^{2} \rho \rho_{\mathrm{air}}\left(\omega \rho-\kappa_{\mathrm{bm}} B\right)^{2}}{\left[A_{\mathrm{ow}} \kappa l_{\mathrm{w}}^{2} \rho\left(H \omega \rho-K_{2} B\right)+A_{\mathrm{a}}^{2} \rho_{\mathrm{air}}^{2}\left(K_{1} \omega \rho-H \kappa_{\mathrm{bm}} \kappa_{\mathrm{cb}} B\right)\right]^{2}}, \\
\tilde{p}_{2, \mathrm{bm}}=\tilde{p}_{2, \mathrm{cb}, b} \frac{4 A_{\mathrm{a}} A_{1} A_{2} \kappa \kappa_{\mathrm{cb}} l_{\mathrm{l}} l_{\mathrm{w}} \rho^{2}\left(\omega \rho-\kappa_{\mathrm{bm}} B\right)\left(A_{\mathrm{a}} l_{\mathrm{a}}^{2} \omega \rho_{\mathrm{air}}+A_{\mathrm{ow}} k l_{\mathrm{w}}^{2} B\right)}{\left[A_{\mathrm{ow}} \kappa l_{\mathrm{w}}^{2} \rho\left(H \omega \rho-K_{2} B\right)+A_{\mathrm{a}} l_{\mathrm{a}}^{2} \rho_{\mathrm{air}}\left(K_{1} \omega \rho-H \kappa_{\mathrm{bm}} \kappa_{\mathrm{cb}} B\right)\right]^{2}}, \\
\tilde{p}_{2 \mathrm{cb}, f}=-\tilde{p}_{2, \mathrm{cb}, b} \frac{2 A_{\mathrm{a}} A_{2} \kappa l_{\mathrm{a}} l_{\mathrm{w}} \rho\left(\omega \rho-\kappa_{\mathrm{bm}} B\right)\left[A_{\mathrm{a}} l_{\mathrm{a}}^{2} \rho_{\mathrm{air}}\left(K_{3} \omega \rho+H \kappa_{\mathrm{bm}} k_{\mathrm{cb}} B\right)+A_{\mathrm{ow}} k l_{\mathrm{w}}^{2} \rho\left(H \omega \rho+K_{4} B\right)\right]}{\left[A_{\mathrm{ow}} \kappa l_{\mathrm{w}}^{2} \rho\left(H \omega \rho-K_{2} B\right)+A_{\mathrm{a}} l_{\mathrm{a}}^{2} \rho_{\mathrm{air}}\left(K_{1} \omega \rho-H \kappa_{\mathrm{bm}} \kappa_{\mathrm{cb}} B\right)\right]^{2}} .
\end{gathered}
$$

In addition to the abbreviations introduced above, we have used the following: $B=A_{\mathrm{rw}} Z_{\mathrm{rw}}, K_{3}=A_{1} k_{\mathrm{bm}}-A_{2} k_{\mathrm{cb}}, K_{4}=A_{1} k_{\mathrm{cb}}-A_{2} k_{\mathrm{bm}}, K_{s}=k_{\mathrm{bm}}+k_{\mathrm{cb}}$, and $K_{d}=k_{\mathrm{bm}}+k_{\mathrm{cb}}$.

\section{References}

1. Pickles, J. O. Introduction to the Physiology of Hearing (Academic Press, 2008).

2. Lighthill, J. Energy flow in the cochlea. J. Fluid Mech. 106, 149-213 (1981).

3. Ulfendahl, M. Mechanical responses of the mammalian cochlea. Progr. Neurobiol. 53, 331-380 (1997).
4. Robles, L. \& Ruggero, M. A. Mechanics of the mammalian cochlea. Physiol. Rev. 81, 1305-1352 (2001).

5. Eguíluz, V. M., Ospeck, M., Choe, Y., Hudspeth, A. J. \& Magnasco, M. O. Essential nonlinearities in hearing. Phys. Rev. Lett. 84, 5232-5235 (2000).

6. Camalet, S., Duke, T., Jülicher, F. \& Prost, J. Auditory sensitivity provided by self-tuned critical oscillations of hair cells. Proc. Natl Acad. Sci. USA 97, 3183-3188 (2000).

7. Hudspeth, A. J., Jülicher, F. \& Martin., P. A critique of the critical cochlea: Hopf-a bifurcation-is better than none. J. Neurophysiol. 104, 1219-1229 (2010).

8. Robles, L., Ruggero, M. A. \& Rich, N. C. Two-tone distortion in the basilar membrane of the cochlea. Nature 349, 413-414 (1991).

9. Robles, L., Ruggero, M. A. \& Rich, N. C. Two-tone distortion on the basilar membrane of the chinchilla cochlea. J. Neurophysiol. 77, 2385-2399 (1997).

10. Cooper, N. P. Harmonic distortion on the basilar membrane in the basal turn of the guinea-pig cochlea. J. Physiol. 509, 277-288 (1998).

11. Jülicher, F., Andor, D. \& Duke, T. Physical basis of two-tone interference in hearing. Proc. Natl Acad. Sci. USA 98, 9080-9085 (2001).

12. Knight, R. D. \& Kemp, D. T. Indications of different distortion product otoacoustic emission mechanisms from a detailed $f_{1}, f_{2}$ area study. J. Acoust. Soc. Am. 107, 1513-1525 (2000).

13. Knight, R. D. \& Kemp., D. T. Wave and place fixed DPOAE maps of the human ear. J. Acoust. Soc. Am. 109, 1513-1525 (2001).

14. Zweig, G. \& Shera., C. A. The origin of periodicity in the spectrum of evoked otoacoustic emissions. J. Acoust. Soc. Am. 98, 2018 (1995).

15. Shera, C. A. \& Guinan, J. J. Evoked otoacoustic emissions arise by two fundamentally different mechanisms: A taxonomy for mammalian OAEs. J. Acoust. Soc. Am. 105, 782-798 (1999).

16. Kalluri, R. \& Shera, C. A. Distortion-product source unmixing: A test of the two-mechanism model for DPOAE generation. J. Acoust. Soc. Am. 109, 622 (2001).

17. Dong, W. \& Olson, E. S. Supporting evidence for reverse cochlear travelling waves. J. Acoust. Soc. Am. 123, 222 (2008).

18. Meenderink, S. W. F. \& van der Heijden, M. Reverse cochlear propagation in the intact cochlea of the gerbil: Evidence for slow travelling waves. $J$. Neurophysiol. 103, 1448-1455 (2010).

19. Ren, T. Reverse propagation of sound in the gerbil cochlea. Nat. Neurosci. 7, 333-334 (2004).

20. Hea, T. W., Nuttall, A. L. \& Ren, T. Two-tone distortion at different longitudinal locations on the basilar membrane. Hear. Res. 228, 112 (2007).

21. He, W., Fridberger, A., Porsov, E., Grosh, K. \& Ren, T. Reverse wave propagation in the cochlea. Proc. Natl Acad. Sci. USA 105, 2729-2733 (2008),

22. Reichenbach, T., Stefanovic, A., Nin, F. \& Huspeth, A. J. Waves on Reissner's membrane: a mechanism for the propagation of otoacoustic emissions from the cochlea. Cell Rep. 1, 374-384 (2012).

23. Tonndorf, J. In Handbook of Sensory Physiology. Auditory System Vol 5 (eds de Boer, E. et al.) 37-48 (Springer, 1976).

24. Bekesy., G. v. Zur theorie des Hörens bei der Schallaufnahme durch Knochenleitung. Ann. Phys. 13, 111-125 (1932).

25. Herzog, H. \& Krainz, W. Das Knochenleitungsproblem. Theoretische Erwägungen und experimentelle Ergebnisse. Z. Hals Nasen $u$. Ohrenheilkunde 15, 300-313 (1926)

26. Timoshenko, S. P. \& Gere., J. M. Theory of Elastic Stability (McGraw-Hill, 1985).

27. Reilly, D. T. \& Burstein, A. H. The elastic and ultimate properties of compact bone tissue. J. Biomech. 8, 393-405 (1975).

28. Raphael, Y. \& Altschuler, R. A. Structure and innervation of the cochlea. Brain Res. Bull. 60, 397-422 (2003).

29. Spatz, H.-Ch., O’Leary, E. J. \& Vincent., J. F. V. Young's moduli and shear moduli in cortical bone. Proc. R. Soc. Lond. B 263, 287-294 (1996).

30. Kringlebotn, M. Acoustic impedances at the oval window and sound pressure. J. Acoust. Soc. Am. 108, 1094-1104 (2000).

\section{Acknowledgements}

We would like to thank A.J. Hudspeth and L. Abbott for helpful discussions and the members Center for Theoretical Neuroscience at Columbia University for hospitality (T.T.). This work has been supported by the the Max Planck Society and the Volkswagen Foundation through a Computational Sciences fellowship (to T.T.) and by a Career Award at the Scientific Interface from the Burroughs Wellcome Fund (to T.R.).

\section{Author contributions}

T.T. and T.R. planned the research, analysed the data and wrote the article. The analytical and numerical computations were performed by T.T.

\section{Additional information}

Competing financial interests: The authors declare no competing financial interests. 
Reprints and permission information is available online at http://npg.nature.com/ reprintsandpermissions/

How to cite this article: Tchumatchenko, T. \& Reichenbach, T. A cochlear-bone wave can yield a hearing sensation as well as otoacoustic emission. Nat. Commun. 5:4160 doi: $10.1038 /$ ncomms5160 (2014). (c) (i) This work is licensed under a Creative Commons Attribution 4.0 International License. The images or other third party material in this article are included in the article's Creative Commons license, unless indicated otherwise in the credit line; if the material is not included under the Creative Commons license, users will need to obtain permission from the license holder to reproduce the material. To view a copy of this license, visit http://creativecommons.org/licenses/by/4.0/ 